\title{
Tunable 2D Photonic Crystal Cavities for Cavity Electro-Optomechanics
}

\author{
Martin Winger, Thiago P. Mayer Alegre, Amir H. Safavi-Naeini, Oskar Painter \\ Thomas J. Watson, Sr., Laboratory of Applied Physics, California Institure of Technology, Pasadena, CA 91125, USA. \\ winger@caltech.edu
}

\begin{abstract}
We present and demonstrate a novel electro-opto-mechanical structure based on a slotted waveguide photonic-crystal cavity, in which electrostatics and optics couple simultaneously to the same "phonon" resonance.
\end{abstract}

(C) 2010 Optical Society of America

OCIS codes: (220.4880) Optomechanics; (230.5298) Photonic crystals; (350.4238) Nanophotonics and photonic crystals

Photonic crystal structures have proven proficient in the field of cavity optomechanics due to their small masses compared to macroscopic Fabry-Pérot cavities, their large optomechanical coupling rates, and their inherent potential for integration in chip-scale photonic networks [1]. Here we demonstrate a novel optomechanical crystal system based on a defect formed in a slotted 2D photonic-crystal waveguide [2]. Integration of a capacitive actuator moreover allows for combining optomechancis and electromechanics in this system.
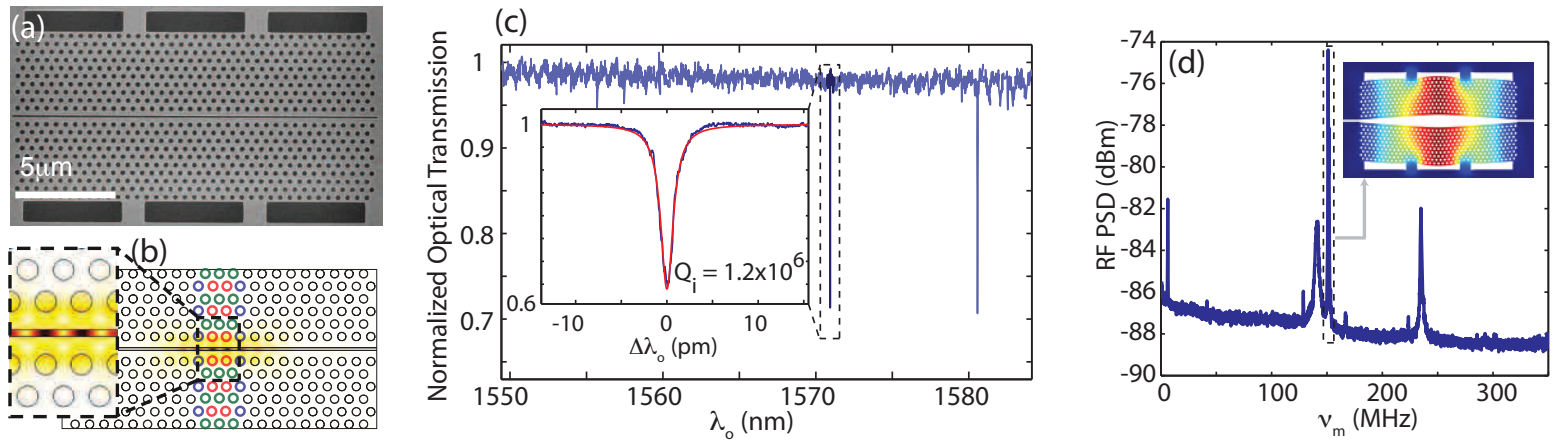

Fig. 1. a, SEM micrograph of a 2D slotted-waveguide defect cavity. b, Cavity electric field intensity distribution $|\mathbf{E}(\mathbf{r})|^{2}$. c, Normalized optical transmission spectrum. Inset: Transmission spectrum for the first order mode with $Q_{i}=1.2 \times 10^{6} \mathbf{d}$, RF modulation spectrum of the transmitted laser indicating a series of reonances correponding to mechanical modes. Inset: Displacement field $|\mathbf{Q}(\mathbf{r})|$ of the fundamental mechanical mode.

Figure 1a shows an SEM micrograph of our photonic crystal device. Starting from a W1 waveguide formed by a row of missing holes in a triangular lattice of air holes in a Silicon slab, a defect is created in its center by local modulation of the lattice constant. Insertion of an air slot in the waveguide allows for significant localization of the cavity field in the slot region, as indicated in Fig. 1b, which shows the result of a 3D finite-element (FEM) simulation of the cavity field distribution. As a result, the frequency of the cavity mode is strongly dependent on the slot width. Mechanical vibrations of the structure that modulate the slot width therefore result in a significant modulation of the cavity frequency. This can be observed by measuring the radiofrequency modulation spectrum of a probe laser locked to a flank of the cavity.

The inset in Fig. 1d shows the mode profile of the fundamental mechanical mode, as obtained from a 3D FEM simulation, which has a frequency of $146.1 \mathrm{MHz}$. Due to the strong mechanical displacement of the slot boundary, this mode results in a large theoretical optomechanical coupling of $g_{\mathrm{OM}}=\partial \omega_{0} / \partial x=2 \pi \times 480 \mathrm{GHz} / \mathrm{nm}$.

Experimentally, the photonic crystal structures were fabricated from Silicon-On-Insulator material using a combination of e-beam lithography and dry- and wet-etching recipes. The devices were tested by resonant spectroscopy using a tapered-fiber based near-field probe [3]. Figure 1c shows the fiber transmission spectrum, indicating two sharp 


\section{CThJ4.pdf}

resonances. The fundamental mode at $\lambda \approx 1570 \mathrm{~nm}$ exhibits an intrinsic $Q$-factor of $Q_{i}=1.2 \times 10^{6}$. Fig. $1 \mathrm{~d}$ shows the power spectral density of the intensity fluctuations of a transmitted probe laser, with its peaks corresponding to different mechanical modes of the structure. The most prominent peak at $151 \mathrm{MHz}$ can be attributed to the fundamental in-plane mode shown in the inset of Fig. 1d which exhibits an optomechanical coupling of $g_{\mathrm{OM}}=2 \pi \times 140 \mathrm{GHz} / \mathrm{nm}$. Given the cavity linewidth of $\kappa=2 \pi \times 160 \mathrm{MHz}$, this mode is in the sideband resolved regime. The other peaks in Fig. 1d correspond to higher order in-plane and out-of-plane modes.

Having a cavity that is optomechanically coupled to the in-plane mechanical mode straightforwardly suggests a useful tuning mechanism for the cavity mode: the slot widh $s$ can be increased by pulling the two photonic crystal membranes apart using a capacitive actuator, thus resulting in a blue-shift of the cavity mode frequency according to $\Delta \omega_{c}=g_{\mathrm{OM}} \Delta s$. As can be seen in the SEM micrograph in Fig. 2a, the capacitor is implemented by two gold wires,
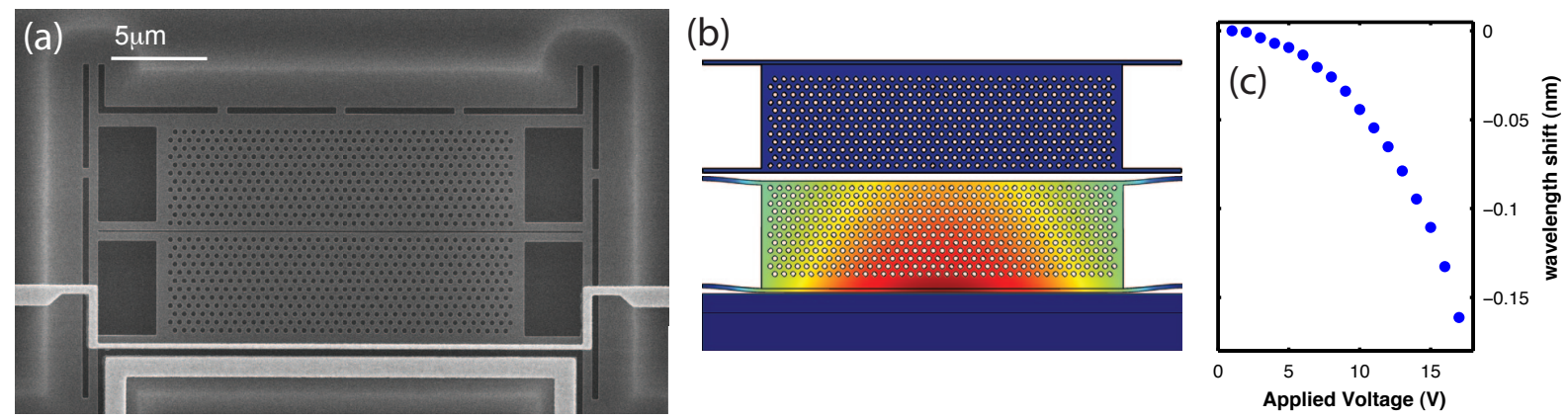

Fig. 2. a, SEM micrograph of a slotted cavity with a capacitive actuator. b, FEM-simulation of the deformed structure (exaggerated) under the influence of $F_{\mathrm{el}}$. c, Experimental tuning curve showing the fundamental cavity-mode wavelength shift as a function of applied voltage.

one of which is deposited on the lower edge of the moveable lower cavity membrane, while the other one is fixed on bulk Silicon. Applying a voltage $V_{\mathrm{DC}}$ across the capacitor results in a pulling force of $F_{\mathrm{el}}=(1 / 2)(d C / d x) V_{\mathrm{DC}}^{2}$ that leads to an increase of the slot width. Figure $2 b$ shows the result of a electro-mechanical FEM simulation that shows the capacitive actuation of the tuning mode of the structure in an exaggerated fashion. These simulations allow us to estimate typical capacitances to be $C \approx 0.3 \mathrm{fF}$, resulting in a tuning force of $F_{\mathrm{el}} \approx 500 \mathrm{pN} / \mathrm{V}_{\mathrm{DC}}^{2}$ for typical device dimensions. The tuning range is determined by the balance between $F_{\mathrm{el}}$ and the restoring spring force, which can be engineered by adjusting the geometry of the membrane suspensions.

Devices like the one shown in Fig. 2a were fabricated in a two-step lithography process in which the cavity pattern is aligned to a pre-defined metal structure. Figure $2 \mathrm{c}$ shows a typical tuning curve of such a device. For a maximum applied DC-voltage of $V_{\mathrm{DC}}=17 \mathrm{~V}$ the cavity could be moved by $\Delta \lambda=-0.16 \mathrm{~nm}$ while maintining a typical $Q$-factor on the order of $Q_{i} \approx 10^{5}$. For devices with less rigid suspensions, tuning up to $2 \mathrm{~nm}$ could be shown.

Given the small cavity linewidths on the order of several picometers, this system allows for the implementation of narrow-band single-mode electrically tunable optical filters. Moreover, the low RC constant of the circuit allows for resonant driving of mechanical modes of the structure by purely electrical means, which e.g. allows for the construction of optical modulators. In addition to these technological applications, the system could prove benefitial for the study of coupled cavity-arrays, where the mutual resonance of individual microcavities is crucial, yet hard to achieve. Finally, the combination of electro- and optomechanics provides a means to tie together radio-frequency and optical photons by their simultaneous coupling to a common "phonon" bus [4].

\section{References}

1. M. Eichenfield, R. Camacho, J. Chan, K. J. Vahala, and O. Painter, "A picogram- and nanometre-scale photoniccrystal optomechanical cavity," Nature 459, 550-556 (2009).

2. A. H. Safavi-Naeini, T. P. Mayer Alegre, M. Winger, and O. Painter, "Optomechanics in an ultrahigh- $Q$ twodimensional photonic crystal cavity," Appl. Phys. Lett. 97, 181106 (2010).

3. C. P. Michael, M. Borselli, T. J. Johnson, C. Chrystal, and O. Painter, "An optical fiber-taper probe for waferscale microphotonic device characterization," Opt. Express 15, 4745 (2007).

4. A. H. Safavi-Naeini and O. Painter, "Proposal for an Optomechanical Travelling Wave Phonon-Photon Translator," arxiv:1009.3529, to appear in New J. Phys. 